\title{
Yet a Faster Motion Estimation Algorithm with Directional Search Strategies
}

\author{
Ying Zhang+*, Wan-Chi Siu* and Tingzhi Shen+* \\ ${ }^{+}$Department of Electronic Engineering \\ Beijing Institute of Technology \\ Beijing, 100081, China \\ *Depart of Electronic and Information Engineering \\ The Hong Kong Polytechnic University \\ Hung Hom, Kowloon, Hong Kong, China
}

\begin{abstract}
An efficient fast search motion estimation algorithm is highly desired in many video coding applications. Most previous fast algorithms are performed in a blind way, without critically making use of the characteristics of motion information of the sequence being coded. In this paper, we propose a new fast search motion estimation algorithm, which uses the directional information of the selected checking points obtained in the search procedure to guide the search. The statistical information which features the motion activities of the blocks in the previous frame is used to predict the characteristics of the motion activities of the blocks in the current frame. This is to save the computational cost and to avoid spending effort on the blocks which will unlikely provide the optimal match. Hence, the computational resource can be re-assigned to locations that deserve to be searched more than others. Extensive experimental work has been done, results of which show that our approach gives a speedup of 5.39 to 1.14 times over that of the recent fast algorithms and 150 times over the exhaustive full search algorithm on average, with a negligible degradation in peak signalto-noise ratio (PSNR).
\end{abstract}

Index Terms - Video Coding, Motion estimation, Motion Vector, Directional Search, and Early termination

\section{INTRODUCTION}

Block based motion estimation is an important part of many standards, such as the MPEG-1,2,4 and the H.261/H.263/H.264, for its noticeable effect on eliminating the temporal redundancy and its simplicity in decoding process. The computational complexity of the full search approach is excessively too high for the implementation of real time encoders. Hence, Many kinds of fast motion estimation algorithms are now available. Typical fast algorithms available in the literature can be grouped into four catalogues: (a) fast full search[1-2], (b) fast search by patterns, such as the Two Dimensional Logarithmic Search (2D-LOG) [3], Three Step Search Algorithm (TSS) [4], Conjugate Directional Search Algorithm (CDS) [5], Cross Search Algorithm (CSA) [6],

Acknowledgement: This research work is a joint research collaboration between the Beijing Institute of Technology (BIT) and The Hong Kong Polytechnic University (PolyU), and is supported by the CERG grant (PolyU5268/06E) of the Hong Kong SAR Government and the Centre for Signal Processing of the PolyU. and Diamond Search (DS)[7], (c) fast search by schemes, such as the Block based Gradient Descent Search (BBGDS)[8], Fast Feature Search[9], Motion Vector Field Adaptive fast Search Technique (MVFAST)[10], and Fast Adaptive Motion Estimation (FAME)[11] algorithm, and (d) Pixel decimation algorithms[1213].

The efficiency of an algorithm can be improved gradually by saving the computational resource on the low motion activity blocks according to the motion information of the nearby regions in the previous and current frames. Is there any possibility to accelerate it further? Since moving objects and the image features are different in diverse video sequences, degradation is always accompanied with a reduction of the computational complexity without using additional prior knowledge of the video sequence being coded. Fortunately, we can obtain some valuable information available in the search procedure. The motion direction obtained in the search procedure and the statistics of the motion information of the blocks in the previous frame can guide the search. In this paper, we propose a motion estimation algorithm by using such information. The new algorithm reduces the search points of the traditional fast search algorithms significantly, while it has a small cost on performance comparing with the full search algorithm.

\section{ALGORITHM DEVELOPMENT}

\subsection{Search Strategies}

In our approach, the search direction obtained in the search procedure is a great help, and we also assume that the matching error surface is unimodal over the search window.

2.1.1 Analysis of the search direction: There are two kinds of directional information, which can be used in the search procedure: the latest directional information and the general directional information.

The latest directional information: We initially assume a simple search procedure as shown in fig. 1 , in which the search pattern is a diamond-shaped pattern. The checking points of the search pattern are examined one by one in the order from the center point, and its points on the left, right, top and bottom. If the best match is found from one of these four surrounding points, it becomes the new center of the pattern. If the center point gives the best match, the center point is the search result. This point is then entitled final point. The three steps as shown in fig. 1 correspond to three movements of the search pattern. 
In each step, if the SAD of the current checking point is smaller, the SAD of this point is selected as the current minimum SAD and the direction of this point with reference to the center of the search pattern is stored in a set, for which let us call it the "search direction set". The search direction set is used to collect the directions of better matching blocks in a step. For example, in the third step of the search procedure as shown in fig.1, the right point is identified, for which the SAD value is 982 and the SAD values of the top and bottom points are both bigger than 982 . The right point is selected for providing a better match, the position is selected to be currently the best position, and the "right" direction is stored in the search direction set. The directional information of the top and bottom points is not collected. However, if one of them were smaller than 982, the direction of this point would also be collected into the directional set. This means that more than one direction may be collected in the search direction set.

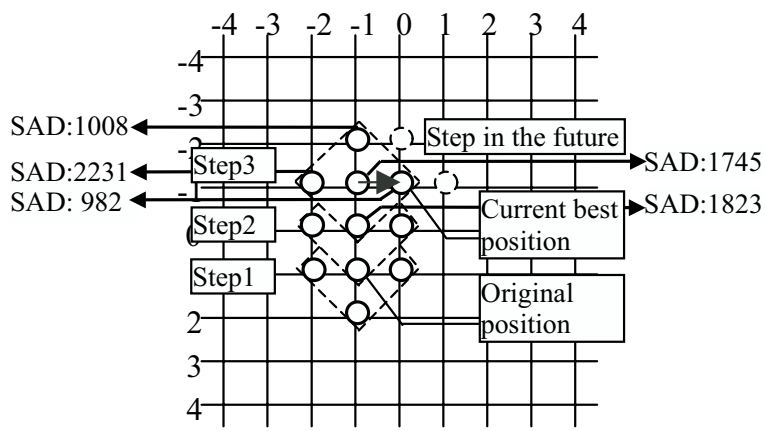

Figure 1. The latest directional vectors

From our extensive experimental work, we have found that there is a high correlation between the direction (stored in the search direction set) of a search point with small SAD and the displacement between the final point and the original point. For example, if a checking point with smaller SAD is found on the right in one step, the final point is more likely to be on the right side of the starting point, as shown in fig. 1. The direction collected in the nearest previous step could imply the latest moving trend of the search pattern, and let us denote it as the latest directional vector, $\mathrm{MV}_{\text {LatDir }}$ (the thick arrow in fig.1):

$$
M V_{\text {LatDir }}=P_{\text {Dirlast }}-P_{\text {centre }}
$$

where $P_{\text {centre }}$ is the position of the center of the search pattern (now the diamond ), and $\mathrm{P}_{\text {Dirlast }}$ is the position corresponding to the better matching points in the search pattern. They are obtained in the latest previous search step.

The general directional information: The displacement of currently the best position and the original position has also a high correlation with that of the final motion vector. For example, if the current best position is on the top-right side of the original position, it is most likely that the final position of the pattern search procedure is on the same side of the original position, as shown in fig. 2. This displacement indicates the general moving trend of the search pattern, namely the general directional information. Let $\mathrm{MV}_{\mathrm{GenDir}}$ (the thick arrow in fig.2) represent the general directional vector; then we have

$$
M V_{\text {GenDir }}=P_{\text {tmp }}-P_{\text {pred }}
$$

where $\mathrm{P}_{\mathrm{tmp}}$ is currently the best position just obtained now, $\mathrm{P}_{\text {pred }}$ is the original position. Subscript 'pred' is used to indicate the "predicted" position since the motion vectors of the neighboring blocks are used to predict the motion vector of the current block before the pattern search stage in this algorithm, the original vector is corresponding to the best-predicted motion vector. By using the latest directional information and the general directional information, we can assist the estimation algorithm and break away from the restriction of the search pattern only.

2.1.2 Search Patterns: In our algorithm, two kinds of search patterns are used for blocks with different characteristics of the motion activities.

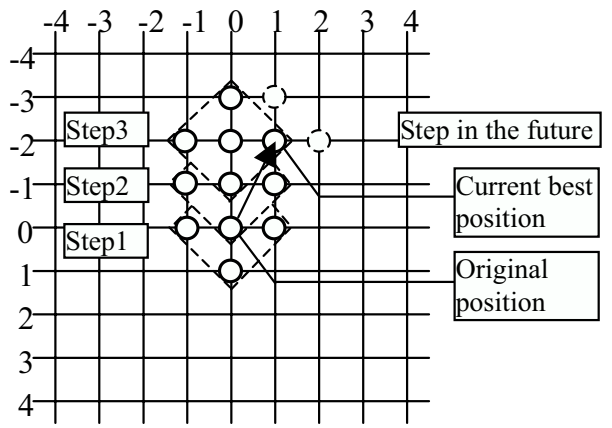

Figure 2. The general directional vectors

Diamond-shaped search pattern, named as pattern1, is initially used, which contains 5 checking points as shown in fig. 3 .

Step1: The search starts from the original point and its four surrounding points. These points are examined one by one (left, right, top, bottom). The SAD of the original point is 1029 . The SAD of the left point is larger than 1029. The SAD of the right point is 938 , which is smaller than the current minimum SAD, is stored as the minimum SAD. This point is entitled currently the best position. And its direction, the "right" direction is stored in the search direction set. After the top and bottom points are examined, the SAD of the right point is still found to be the smallest. Hence, we select this point as the new center of the search pattern.

Step2: In step1, we have collected the "right" direction in the search direction set, which is the latest directional information. Note that in this step, the latest directional information is also the same as the general directional information, since the general directional information is obtained from currently the best position $\mathrm{P}_{\text {tmp }}$ (the new center point of the search pattern in this step) and the original position $\mathrm{P}_{\text {pred }}$ (the point in the Original position). The latest directional vector can be used to select the checking points in the search pattern as shown in fig. 3 . The point with a circle is selected to be a candidate in step2.

According to the directional information, which is collected in step1, we do not have sufficient information whether the best match is going to be on the top or on the bottom of the new centre. Hence, both the checking points on the top and bottom are selected to be candidates as shown in fig.3 (points with black dots). Actually, in this step we have to examine 3 checking points, this number is just the same as that needed by the diamond-based search. Due to the space limit, let us skip step3, and illustrate further the idea in step4.

After step3, the current minimum SAD is 876 , the latest directional MVs (stored in the direction set) are pointing at the "right" and 'top' directions, and the general directional MV is projected onto the $+x$-axis and $-\mathrm{y}$-axis. Hence, in step 4, the right point and the top point are selected as the candidates and examined. 
The SAD calculation for the checking point on the left is saved, comparing with the diamond-based search algorithm. This saving point is marked with a cross " $\times$ " as shown in fig. 3 .

For the blocks, the motion vector of which is more likely to be different with the surrounding blocks or the collocated block in the previous frame, we apply another pattern. It is essentially a block based search pattern, and contains 9 checking points. The process of search pattern2 is similar to that of search pattern1.

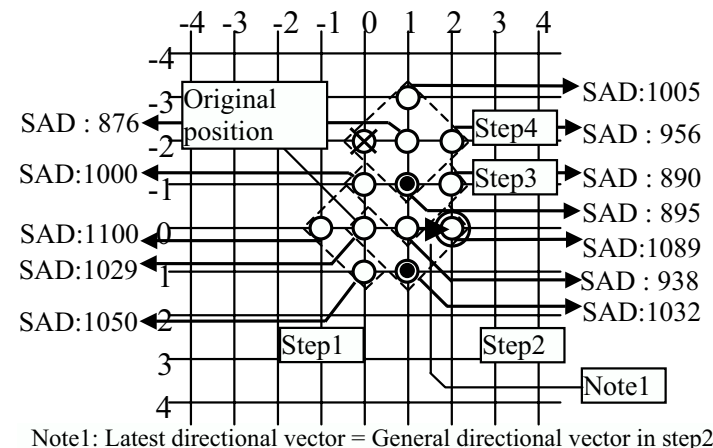

Figure 3. Sample Search Procedure.

\subsection{Block classification according to the statistical motion information}

In this paper, we introduce our idea that the motion vectors of the neighboring blocks are used to predict the motion vector of the current block before the pattern search stage. If the increase of the motion vector of a block in the pattern search stage is not zero, it means that the best motion vector is not the same as the predicted motion vector. The prediction process of this block has failed to predict exactly the motion vector of this block. This kind of blocks are classified into the special block group (Group S), whereas the other blocks are classified into ordinary block group (Group O)

The special blocks and the ordinary blocks of the current frame can be "prejudged" according to the block classification of the previous frame. Our process is to set the special blocks of the previous frame and their surrounding 8 blocks as the prejudged special blocks of the current frame, and set the other blocks as prejudged ordinary block. There is a higher probability for a block in the prejudged special block group to have a motion vector different from its neighboring blocks than a block in the prejudged ordinary block. In order to save computational effort, we suggest dealing with prejudged special blocks with pattern2 and dealing with ordinary blocks differently, with pattern1.

\subsection{Overall structure of algorithm}

Fig. 4 shows a flow chart of the present approach. Initially, blocks in a frame have to be classified into prejudged special group and prejudged ordinary group according to the statistical motion information of the previous frame (See section 2.2).

Essentially we must find the SAD of the initial location of the block. After checking this point, we are going to find a new centre of the search window, which should be able to enhance the centrebiased feature of our algorithm. This is achieved by checking the points corresponding to the predicted motion vectors obtained from the neighboring blocks in the current frame and the co-located locations of the previous frame. Let $t$ indicate the frame number and $(\mathrm{i}, \mathrm{j})$ represents the position of the block. We then have to find the SADs using (i) the motion vectors of the adjacent blocks, $\operatorname{MV}(i-1, j, t), M V(i, j-1, t)$ and MV(i+1,j-1,t) in the current frame, and (ii) the medium MV of the above three MVs, and (iii) the motion vectors of $(M V(i, j, t-1)$ and $M V(i+1, j+1, t-1)$ in the previous frame. The one with the least SAD is chosen as the centre of the search window.

Blocks in the prejudged special group and blocks in the prejudged ordinary group are dealt with differently in the subsequent process. If the current block is a prejudged special block, we use pattern 2 (with 9-pixel block) for the search of the current block. Otherwise, we use the pattern 1 (5-pixel diamond shape) to do the search for the current block.

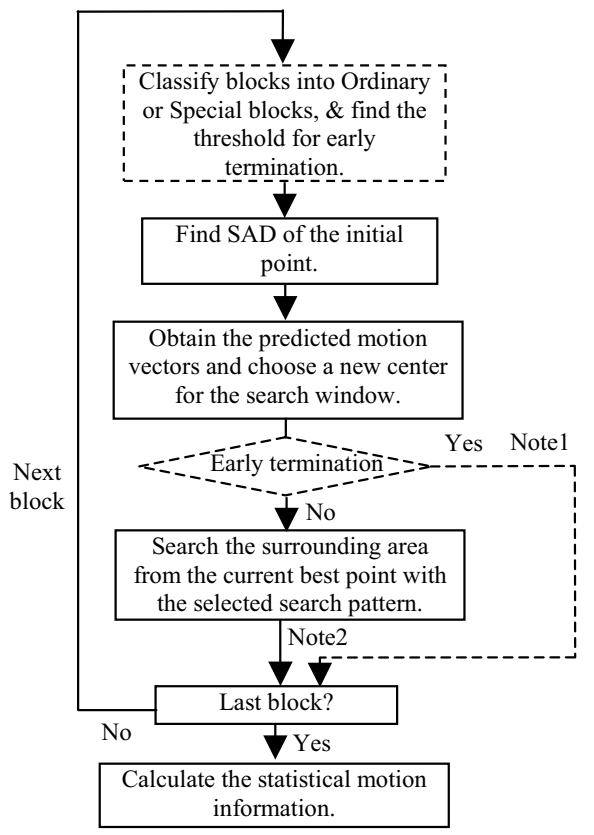

Note1: $\mathrm{MV}=$ predicted $\mathrm{MV}$, for $\mathrm{SAD}<$ threshold Note2: $\mathrm{MV}=$ predicted $\mathrm{MV}+\Delta \mathrm{MV}$

Figure 4. Flow charts of the suggested algorithm

Early termination: The motion estimation results of the prejudged ordinary blocks are probably the same with their neighboring blocks. Hence, an early termination is applied to the prejudged ordinary blocks, for which the early termination is executed if the SAD value of the best-predicted motion vector is lower than a threshold. The threshold is determined according to the percentage of the ordinary blocks of the previous frame and the probability distribution of different SADs values between the minimum and maximum of the previous frame.

In the pattern search stage, the actual MV is obtained by searching the area surrounding currently the best predicted point. The increase of MV generated in this part is labelled as $\Delta \mathrm{MV}$. After searching for the best match of all the blocks in the current frame, the statistical motion information is obtained for uses in next frame.

\section{EXPERIMENTAL RESULTS}

Performance of the present algorithm is compared with several common fast search algorithms available in the literature. The 
Block Based Gradient Descent Search, Diamond Search and the Conjugate Directional Search are three traditional search algorithms that are most similar with the present algorithm. The Three-step search is supposed to be an early algorithm, but it is the simplest algorithm. The MVFAST is a widely used algorithm, whilst the FAME is a newly proposed algorithm.

The PSNR (PSNR) listed in the Table I is the average PSNR value of the luminance component of the decoded frames. SpUp is the speed-up on the average number of SAD checking points as compared with the full search algorithm. SpUp is the average speed up on real time measurement as compared with the full search algorithm. The software was the XVID encoder. We used a PC with Inter(R) Pentium (R) D, 3.60GHz CPU and 1GB RAM. A large number of sequences with the CIF format were tested. For limited space, only three sequences with low, medium and high motion activities are shown in table I. The block size was $16 \times 16$. The size of the search window was $32 \times 32$. The bit rate was set to $380000 \mathrm{bits} / \mathrm{sec}$.

Table I: Performance comparison of different algorithms, at a bitrate $=380 \mathrm{~K} \mathrm{bit} / \mathrm{sec}$.

\begin{tabular}{|l|c|c|c|c|c|c|c|c|c|}
\hline Sequences & \multicolumn{3}{|l|}{ Hall } & \multicolumn{3}{l|}{ Container } & \multicolumn{2}{c|}{ Flower } \\
\hline & PSNR & SpUp & SpUp $_{\mathrm{t}}$ & PSNR & SpUp & SpUp & PSNR & SpUp & SpUp \\
\hline FS & 37.21 & 1 & 1 & 35.64 & 1 & 1 & 23.87 & 1 & 1 \\
\hline TSS & 37.22 & 31 & 27 & 35.58 & 31 & 28 & 23.36 & 31 & 25 \\
\hline DS & 37.26 & 70 & 62 & 35.57 & 75 & 68 & 23.91 & 59 & 53 \\
\hline BBGDS & 37.25 & 94 & 80 & 35.58 & 103 & 79 & 23.92 & 73 & 67 \\
\hline CDS & 37.27 & 181 & 158 & 35.52 & 192 & 155 & 23.86 & 151 & 128 \\
\hline MVFAST & 37.28 & 147 & 145 & 35.56 & 170 & 134 & 23.95 & 109 & 92 \\
\hline FAME & 36.64 & 103 & 80 & 35.00 & 91 & 74 & 24.08 & 89 & 71 \\
\hline OurAlg. & $\mathbf{3 7 . 3 4}$ & $\mathbf{4 4 5}$ & $\mathbf{2 7 3}$ & $\mathbf{3 5 . 7 5}$ & $\mathbf{7 8 6}$ & $\mathbf{3 7 0}$ & $\mathbf{2 3 . 9 6}$ & $\mathbf{1 5 7}$ & $\mathbf{1 1 8}$ \\
\hline
\end{tabular}

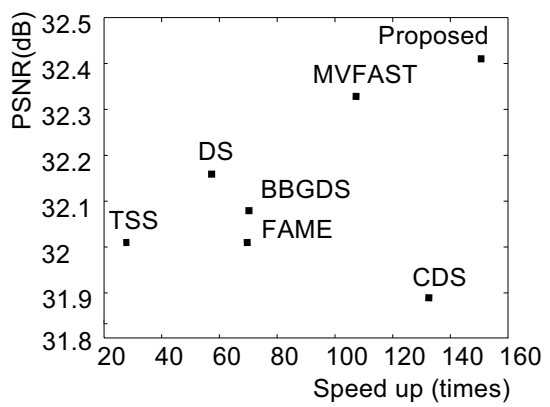

Figure 5. Diagram showing the relative performances of algorithms.

Comparing our algorithm (OurAlg.) with the full search algorithm (FS), the performance of our algorithm is better for all the 3 sequences. The increases of the PSNR (at a bit rate of approximately $380 \mathrm{~K} \mathrm{~b} / \mathrm{s}$ ) vary from $0.09 \mathrm{~dB}$ to $0.13 \mathrm{~dB}$. For some other sequences, the performance of the present algorithm is slightly inferior to the full search algorithm.

The overall performance of our algorithm is usually much better than that of other fast search algorithms; especially it is the fastest one. This fact is clearly indicated in fig 5, where the PSNR and the speed up are the average values obtained by using 18 sequences (claire, akiyo, mother daughter, hall, silent, highway, container, erik, paris, foreman, football, waterfall, coastguard, bus, tempete, Stefan, flower, mobile).

\section{CONCLUSION}

We have done much work on selecting the shape of the search patterns and the way of finding the predictive motion vectors. We eventually arrive at the idea of using the directional information, which is extracted from the observation of the distribution of the candidate checking points and the final points of the search patterns. This algorithm also involves the idea of adaptive search pattern and the characteristics of the motion activities to classify blocks into special and ordinary groups. These rely heavily on the statistical information of the motion characteristics of the successive frames. Results our experimental work show that our approach is fast than all algorithms available in the literature with similar quality and bit-rates. Further work is being done on its further analysis and an extension to sub-pixel motion estimation.

\section{REFERENCES}

[1] C. C. Zhu, W.S. Qi and W. Ser, "Predictive Fine Granularity Successive Elimination for Fast Optimal Block-Matching Motion Estimation," IEEE Transactions on Image Processing, vol.14, no.2, pp.213-221, Feb. 2005.

[2] Ko-Cheung Hui, Wan-Chi Siu and Yui-Lam Chan, "New Adaptive Partial Distortion Search using Clustered Pixel Matching Error Characteristic," IEEE Transactions on Image Processing, vol.14, no.5, pp.597-607, May 2005.

[3] J.R. Jain and A.K. Jain, "Displacement Measurement and Its Application in Interframe Image Coding," IEEE Transactions on Communication, vol. 29, no. 12, pp. 1799-1808, Dec. 1981.

[4] R. Li, B. Zeng, and M.L. Liou, "A new three-step search algorithm for block motion estimation," IEEE Transactions on Circuits and Systems for Video Technology, vol. 4, no. 4, pp. 438-442, Aug. 1994.

[5] R. Srinivasan and K. Rao, "Predictive coding based on efficient motion estimation," IEEE Transactions on Communications, vol. 33, no. 8, pp. 888-896, Aug. 1985.

[6] M. Ghanbari, "The Cross-Search Algorithm for Motion Estimation," IEEE Transactions on Communications, vol. 38 no. 7, July 1990.

[7] S. Zhu and K.K. Ma, "A New Diamond Search Algorithm for Fast Block Matching," IEEE Transactions on image processing, vol. 9, no. 2, pp. 287-290, Feb. 2000

[8] L.K. Liu and E. Feig, "A Block-based Gradient Descent Search Algorithm for block-based motion estimation in video coding," IEEE Transactions on Circuits and Systems for Video Technology, vol. 6, no. 4, pp. 419-422, Aug. 1996

[9] Yui-Lam, Chan and Wan-Chi Siu, "An Efficient Search Strategy for Block Motion Estimation using Image Features," IEEE Transactions on Image Processing, vol.10, no.8, pp.1223-38, Aug. 2001.

[10] Prabhudev Irappa Hosur and Kai-Kuang Ma, "Motion Vector Field Adaptive Fast Motion Estimation," Second International Conference on Information, Communications and Signal Processing (ICICS '99), December 1999 .

[11] I. Ahmad, Zheng. Weiguo, Luo. Jiancong, Liou. Ming, "A Fast Adaptive Motion Estimation Algorithm", IEEE Transactions on Circuits and Systems for Video Technology, vol.16, no 3, pp.420438, Mar. 2006.

[12] Bede Liu and André Zaccarin, "New Fast Algorithm for the Estimation of Block Motion Vectors," IEEE Transactions on Circuits and Systems for Video Technology, vol. 3, no. 2, pp. 148-157, Apr. 1993.

[13] Yiu-Lam Chan and Wan-Chi Siu, "New Adaptive Pixel Decimation for Block Motion Vector Estimation," IEEE Transactions on Circuits and Systems for Video Technology, pp.113-118, vol.6, no.1, Feb. 1996. 